УДК 621.315 .592

\title{
Эффекты МакКарди в теплопроводности упруго анизотропных кристаллов в режиме кнудсеновского течения фононного газа
}

\author{
(С) И.Г. Кулеев, И.И. Кулеев, С.М. Бахарев \\ Институт фризики металлов имени М.Н. Михеева Уральского отделения Российской академии наук, \\ 620990 Екатеринбург, Россия \\ E-mail: kuleev@imp.uran.ru
}

(Получена 26 июля 2017 г. Принята к печати 20 декабря 2017 г.)

Исследовано влияние фокусировки фононов на фононный транспорт в полупроводниковых и диэлектрических кристаллах с различным типом анизотропии упругой энергии при низких температурах. Рассчитаны эффекты МакКарди в теплопроводности упруго анизотропных кристаллов в режиме кнудсеновского течения фононного газа. Проанализировано влияние фокусировки фононов на распространение и граничное рассеяние фононов в образцах с квадратным и прямоугольным сечениями, и дано физическое объяснение эффектам МакКарди.

DOI: $10.21883 /$ FTP.2018.13.46864.8690

\section{1. Введение}

Анизотропия упругих свойств кристаллов приводит к ряду новых эффектов в фононном транспорте [1-4]. Одним из таких эффектов является фокусировка фононов. В работах [2-4] показано, что из-за неколлинеарности фазовой и групповой скоростей фононный поток, излучаемый точечным источником тепла, фокусируется вдоль определенных направлений в кристаллической решетке. Экспериментальные исследования, проведенные в работе [1], показали, что при низких температурах, когда доминирует диффузное рассеяние фононов на границах, фокусировка фононов приводит к двум эффектам в теплопроводности кубических кристаллов (эффекты Мак Карди). Первым эффектом является зависимость теплопроводности образцов $\mathrm{Si}$ с квадратным сечением от направления градиента температуры относительно кристаллографических осей: при температуре $T=3 \mathrm{~K}$ величина теплопроводности в направлении [001] оказалась на 40 и 50\% больше, чем в направлениях [011] и [111] соответственно. Для кристаллов $\mathrm{CaF}_{2}$, напротив, в направлении [111] теплопроводность оказалась на 28 и 40\% больше, чем в направлениях [011] и [001] соответственно. Вторым эффектом Мак Карди [1] является зависимость величин теплопроводности образцов с прямоугольным сечением при низких температурах от ориентации боковых граней. Для двух исследованных в [1] кристаллов $\mathrm{Si}$, имеющих одинаковые геометрические параметры, при одинаковом направлении градиента температуры [110] оказалось, что теплопроводность $(\kappa)$ образца с широкой гранью $\{001\}$ и узкой $\{110\}$ на $33 \%$ выше, чем для ориентации широкой грани $\{110\}$ и узкой $\{001\}$. При температурах выше максимума $\kappa(T)$ длина свободного пробега фононов становится меньше поперечных размеров образца, и теплопроводность становится изотропной - она не зависит от направления потока тепла в кристалле.

В рамках теории Казимира-МакКарди [1,5] для образцов конечной длины в работах [6-8] получены ана- литические выражения для времен релаксации фононов при диффузном рассеянии на границах. Эти решения позволили в [7] рассчитать оба эффекта в теплопроводности кристаллов $\mathrm{Si}$ и согласовать температурные зависимости и анизотропию теплопроводности образцов с квадратным и прямоугольным сечениями с экспериментальными данными [1] во всем исследованном интервале температур. В интервале от 3 до $15 \mathrm{~K}$ температурные зависимости теплопроводности для всех образцов $\mathrm{Si}$, рассчитанные в [7] при учете граничного и изотопического рассеяния, согласуются с экспериментальными данными [1] в пределах погрешности эксперимента без использования подгоночных параметров. Поскольку эффекты Мак Карди [1], характеризующие анизотропию теплопроводности, при низких температурах для кристаллов $\mathrm{Si}$ и $\mathrm{CaF}_{2}$ достигают 50 и 40\%, то для широкого круга исследователей большой интерес представляют величины этих эффектов в других упруго анизотропных кристаллах. Поэтому мы рассчитали эффекты Мак Карди для всех наиболее актуальных полупроводниковых и диэлектрических кристаллов с различным типом анизотропии упругой энергии при достаточно низких температурах, когда реализуется режим кнудсеновского течения фононного газа. Эта проблема является актуальной, поскольку ранее эффекты, обусловленные фокусировкой фононов, не учитывались, а для граничного рассеяния в монокристаллических образцах использовались модель изотропной среды и теория Казимира [5]. При этом зависимости теплопроводности от направлений теплового потока и геометрических параметров образцов учитывались введением подгоночных параметров [9-11]. Следует отметить, что при изложении экспериментальных результатов в обзорах [12-14] также использовалась модель изотропной среды: не указывались направления теплового потока и ориентации плоскостей пленок относительно кристаллографических осей. В [15] показано, что изменение ориентации кремниевой пленки может приводить к изменению значений теплопроводности более чем в 2 раза. 
Второй проблемой настоящей работы является объяснение физических причин, приводящих к эффектам Мак Карди. Наиболее необычным является второй эффект Мак Карди. Почему для одного и того же направления теплового потока [110] изменение ориентации широкой грани образца приводит к значительному изменению теплопроводности?

При интерпретации ориентационной зависимости теплопроводности пленок в работе [16] предполагалось, что плоскости пленок, имеющие различную ориентацию, обладают различной рассеивающей способностью. Эта гипотеза является некорректной. Дело в том, что феноменологическая теория Казимира-Мак Карди [1,5] основана на предположении, что все фононы при соударении с поверхностью поглощаются, а затем переизлучаются изотропно по направлению внутрь образца. Поэтому в каждой точке поверхности независимо от ее ориентации фононы всех поляризаций рассеиваются диффузно. Таким образом, рассеивающая способность, или интенсивность рассеяния, плоскостей с различной ориентацией одинакова. Поэтому мы рассмотрели влияние анизотропии упругой энергии на граничное рассеяние фононов в образцах с прямоугольным сечением и показали, что зависимость теплопроводности от ориентации широкой грани образца обусловлена изменением распределения теплового потока по поперечному сечению образца из-за фокусировки быстрой поперечной моды в кристаллах обоих типов. При этом медленная поперечная мода, играющая основную роль в первом эффекте Мак Карди, исключается из рассмотрения, поскольку для направления [110] она дефокусируется как в плоскости $\{001\}$, так и в плоскости $\{110\}$. Однако быстрая поперечная мода для волновых векторов в плоскости $\{001\}$ изотропна (эффект фокусировки отсутствует), а в плоскости $\{110\}$ она фокусируется в направлении теплового потока. Следует отметить, что в кубических кристаллах для произвольного направления волнового вектора распространяются квазипоперечные и квазипродольные моды и только для симметричных направлений распространяются чистые моды. В работе [17] проанализирован спектр и вектора поляризации в кубических кристаллах с различным типом анизотропии упругой энергии. Показано, что вклад поперечной компоненты в квазипродольные колебания для них мал и им можно пренебречь. Для квазипоперечных мод продольная компонента не является малой, должна учитываться при анализе поглощения ультразвука и процессах электрон-фононной релаксации и соответственно в термоэдс фононного увлечения. Для кнудсеновского течения фононного газа, в соответствии с [4-7], предполагается, что каждая из акустических мод рассеивается на поверхности диффузно. Нами показано, что для кристаллов с различным типом анизотропии упругой энергии поведение эффектов Мак Карди качественно различается. Этот результат представляет несомненный научный интерес и может быть использован для технических приложений.

\section{2. Теплопроводность упругоанизотропных кристаллов в режиме кнудсеновского течения фононного газа}

Проанализируем фононный транспорт в полупроводниковых и диэлектрических кристаллах кубической симметрии при температурах, гораздо меньших температуры Дебая $\left(T \ll T_{\mathrm{D}}\right)$, когда для фононов применима модель анизотропного континуума. В этой модели анизотропия спектра $\omega_{q}^{\lambda}=S^{\lambda}(\theta, \varphi) q$ определяется фазовой скоростью $S^{\lambda}(\theta, \varphi)$, которая зависит от угловых переменных $\theta$ и $\varphi$ вектора q. Индексы поляризации $\lambda$ выберем следующим образом: индекс $L$ соответствует продольным фононам, $t_{1}$ и $t_{2}-$ „быстрой“ (верхней) и „медленной“ (нижней) поперечным модам в системе координат по ребрам куба, она определена в [17]. Групповая скорость фонона, которая определяет направление переноса энергии, для произвольного направления волнового вектора имеет вид [6]

$$
\begin{gathered}
\mathbf{V}_{g}^{\lambda}(\theta, \varphi)=S^{\lambda}(\theta, \varphi) \tilde{\mathbf{V}}_{g}^{\lambda}(\theta, \varphi), \\
\tilde{\mathbf{V}}_{g}^{\lambda}(\theta, \varphi)=\mathbf{n}+S_{\theta}^{\lambda} \mathbf{e}_{\theta}+S_{\varphi}^{\lambda} \mathbf{e}_{\varphi}, \\
S_{\theta}^{\lambda}(\theta, \varphi)=\left(\frac{1}{S^{\lambda}}\right) \frac{\partial S^{\lambda}}{\partial \theta}, \quad S_{\varphi}^{\lambda}(\theta, \varphi)=\frac{1}{\sin \theta}\left(\frac{1}{S^{\lambda}}\right) \frac{\partial S^{\lambda}}{\partial \varphi} .
\end{gathered}
$$

Здесь $\mathbf{e}_{\theta}=(\cos \theta \cos \varphi, \cos \theta \sin \varphi,-\sin \theta), \mathbf{e}_{\varphi}=(-\sin \varphi$, $\cos \varphi, 0), \quad$ a $\quad \mathbf{n}=\mathbf{q} / q=(\sin \theta \cos \varphi, \sin \theta \sin \varphi, \cos \theta)$ единичный волновой вектор фонона. Вектора $\mathbf{n}, \mathbf{e}_{\theta}$ и $\mathbf{e}_{\varphi}$ образуют взаимно ортогональную тройку единичных векторов.

Проанализируем фононный транспорт в монокристаллических образцах длиной $L$, имеющих прямоугольное сечение со сторонами $D$ (толщина) и $W=\mu D$ (ширина). Для образцов с квадратным сечением параметр $\mu=1$. При температурах, гораздо меньших температуры Дебая $\left(T \ll T_{\mathrm{D}}\right)$, когда теплосопротивление обусловлено диффузным рассеянием фононов на границах, воспользуемся моделью анизотропного континуума и представим теплопроводность в виде аддитивной суммы вкладов от всех акустических мод для направления потока тепла $[I(\psi)]$ и ориентации плоскости $\{J\}$, в которой угол $\psi$ отсчитывается от оси $z$ (см. далее, а также [15]):

$$
\begin{gathered}
\kappa_{[I(\psi)]}^{\{J\}}(T)=\frac{1}{3} C_{V}(T) \bar{S} \Lambda_{[I(\psi)]}^{\{J\}}=\sum_{\lambda} \frac{1}{3} C_{V}^{\lambda}(T) \bar{S}^{\lambda} \Lambda_{[I(\psi)]}^{\lambda\{J\}}, \\
C_{V}=\frac{2 \pi^{2} k_{\mathrm{B}}^{4}}{5 \hbar^{3}} T^{3} \frac{1}{3} \sum_{\lambda}\left\langle\left(S^{\lambda}\right)^{-3}\right\rangle, \\
\bar{S}=\sum_{\lambda}\left\langle\left(S^{\lambda}\right)^{-2}\right\rangle / \sum_{\lambda}\left\langle\left(S^{\lambda}\right)^{-3}\right\rangle, \bar{S}^{\lambda}=\left\langle\left(S^{\lambda}\right)^{-2}\right\rangle\left\langle\left(S^{\lambda}\right)^{-3}\right\rangle, \\
\left\langle\left(S^{\lambda}\right)^{-n}\right\rangle=\frac{1}{4 \pi} \int d \Omega_{q}\left(S_{\lambda}\right)^{-n}, \quad d \Omega_{q}=\sin \theta d \theta d \varphi . \quad(2)
\end{gathered}
$$


Удельные теплоемкости $C_{V}(T)$ и $C_{V}^{\lambda}(T)$, а также средние скорости фонона $\bar{S}$ и $\bar{S}^{\lambda}$ не зависят от направления потока тепла. Поэтому анизотропия теплопроводности и вкладов в нее от акустических мод определяются длинами свободного пробега $\Lambda_{[I(\psi)]}^{\{J\}}$ и $\Lambda_{[I(\psi)]}^{\{J\} \lambda}$. Как видно из формул (2), теплопроводность в режиме кнудсеновского течения фононного газа $\propto T^{3}$, как и теплоемкость трехмерных систем в теории Дебая. Длины свободного пробега для направления потока тепла $[I(\psi)]$ и ориентации широкой плоскости образца $\{J\}$ могут быть представлены в виде $[6,15]$

$$
\begin{gathered}
\Lambda_{[I(\psi)]}^{\{J\}}=\frac{3}{4 \pi} \frac{1}{\sum_{\lambda 1}\left\langle\left(S^{\lambda 1}\right)^{-2}\right\rangle} \sum_{\lambda} \int d \Omega_{q} \frac{I_{[I(\psi)]}^{\lambda\{J\}}(\theta, \varphi)}{\left[S^{\lambda}(\theta, \varphi)\right]^{3}}, \\
\Lambda_{[I(\psi)]}^{\lambda\{J\}}=\frac{3}{4 \pi} \frac{1}{\left\langle\left(S^{\lambda}\right)^{-2}\right\rangle} \int d \Omega_{q} \frac{I_{[I(\psi)]}^{\lambda\{J\}}(\theta, \varphi)}{\left[S^{\lambda}(\theta, \varphi)\right]^{3}} .
\end{gathered}
$$

Релаксационные функции $I_{[I(\psi)]}^{\lambda\{J\}}(\theta, \varphi)$ для образцов с прямоугольным сечением при выполнении неравенств $\mu\left|V_{g 1}^{\lambda}\right|>\left|V_{g 2}^{\lambda}\right|$ и $\left|V_{g 3}^{\lambda} / V_{g 1}^{\lambda}\right| \geq k_{0}=L / 2 D$ или $\mu\left|V_{g 1}^{\lambda}\right|<\left|V_{g 2}^{\lambda}\right|$ и $\left|V_{g 3}^{\lambda} / V_{g 2}^{\lambda}\right| \geq k_{0} / \mu$ определяются выражениями $[6,15]$

$$
\begin{aligned}
I_{[I(\psi)]}^{\lambda\{J\}}(\theta, \varphi)= & \frac{D k_{0}\left|V_{g 3}^{\lambda}\right|}{\mu}\left\{\mu-\frac{k_{0}}{2} \frac{\left(\left|V_{g 2}^{\lambda}\right|+\mu\left|V_{g 1}^{\lambda}\right|\right)}{\left|V_{g 3}^{\lambda}\right|}\right. \\
& \left.+\frac{\left(k_{0}\right)^{2}}{3} \frac{\left|V_{g 1}^{\lambda}\right|\left|V_{g 2}^{\lambda}\right|}{\left(V_{g 3}^{\lambda}\right)^{2}}\right\} .
\end{aligned}
$$

Если $\mu\left|V_{g 1}^{\lambda}\right|>\left|V_{g 2}^{\lambda}\right|$ при $\left|V_{g 3}^{\lambda} / V_{g 1}^{\lambda}\right|<k_{0}$ или $\mu\left|V_{g 1}^{\lambda}\right|<\left|V_{g 2}^{\lambda}\right|$ при $\left|V_{g 3}^{\lambda} / V_{g 2}^{\lambda}\right|<k_{0} / \mu$, то функции $I_{[I(\psi)]}^{\lambda\{J\}}(\theta, \varphi)$ определяются выражениями для образцов бесконечной длины

$$
\begin{gathered}
I_{[I(\psi)]}^{\lambda\{J\}}(\theta, \varphi)=\frac{D}{6 \mu}\left[\left(\frac{V_{g 3}^{\lambda}}{V_{g 1}^{\lambda}}\right)^{2}\left(3 \mu\left|V_{g 1}^{\lambda}\right|-\left|V_{g 2}^{\lambda}\right|\right)\right], \\
\mu\left|V_{g 1}^{\lambda}\right|>\left|V_{g 2}^{\lambda}\right|, \\
I_{[I(\psi)]}^{\lambda\{J\}}(\theta, \varphi)=\mu \frac{D}{6}\left[\left(\frac{V_{g 3}^{\lambda}}{V_{g 2}^{\lambda}}\right)^{2}\left(3 \mu\left|V_{g 2}^{\lambda}\right|-\mu\left|V_{g 1}^{\lambda}\right|\right)\right], \\
\mu\left|V_{g 1}^{\lambda}\right|<\left|V_{g 2}^{\lambda}\right|,
\end{gathered}
$$

Здесь $V_{g 3}^{\lambda}(\theta, \varphi), V_{g 1}^{\lambda}(\theta, \varphi), V_{g 2}^{\lambda}(\theta, \varphi)$ - проекции групповой скорости на направление градиента температуры и перпендикулярные ему направления в системе координат, связанной с образцом.

Для учета влияния фокусировки фононов на анизотропию теплопроводности монокристаллических образцов достаточно выразить ориентационные параметры направление потока тепла $[I(\psi)]$ и ориентацию плоскости $\{J\}$, в которой угол $\psi$ отсчитывается от оси $z,-$ через компоненты групповой скорости. Для этого рассмотрим вращение потока тепла в двух симметричных плоскостях: широкая грань образца совпадает с плоскостью грани куба $y z\{J\}=\{100\}$; широкая грань образца совпадает с диагональной плоскостью $\{J\}=\{110\}$. Определим систему координат, связанную с образцом: ось „3“ направим вдоль оси образца, которая совпадает с направлением теплового потока. Ось „1“ (ось вращения) направим перпендикулярно широкой грани образца или одной из граней для образцов с квадратным сечением. Она определяет ориентацию плоскости $\{J\}$. Ось „““ направим перпендикулярно узкой грани образца. Учтем, что спектр фононов определен в системе координат по ребрам куба. Тогда компоненты групповой скорости фононов в принятой системе координат для рассматриваемых случаев могут быть представлены в виде

$$
\begin{gathered}
V_{g 3}^{\lambda}=-V_{g y}^{\lambda} \sin \psi+V_{g z}^{\lambda} \cos \psi, \\
V_{g 2}^{\lambda}=V_{g y}^{\lambda} \cos \psi+V_{g z}^{\lambda} \sin \psi, \quad V_{g 1}^{\lambda}=V_{g x}^{\lambda}, \\
V_{g 3}^{\lambda}=\left(V_{g x}^{\lambda}+V_{g y}^{\lambda}\right) \sin \psi / \sqrt{2}+V_{g z}^{\lambda} \cos \psi, \\
V_{g 2}^{\lambda}=\left(V_{g x}^{\lambda}+V_{g y}^{\lambda}\right) \cos \psi / \sqrt{2}-V_{g z}^{\lambda} \sin \psi, \\
V_{g 1}^{\lambda}=\left(V_{g x}^{\lambda}-V_{g y}^{\lambda}\right) / \sqrt{2} .
\end{gathered}
$$

Компоненты групповой скорости фононов в декартовой системе координат могут быть определены из формул (1). Зависимость направления теплового потока от угла $\psi$ определяется компонентой $V_{g 3}^{\lambda}$. Проекция компоненты $V_{g 1}^{\lambda}$ не зависит от угла $\psi$, поскольку является осью вращения. Она определяет ориентацию широкой грани образца $\{J\}$. Компонента $V_{g 2}^{\lambda}$ зависит от угла $\psi$ и определяет ориентацию двух других граней образца.

Для расчета первого эффекта Мак Карди необходимо определить ориентационные параметры для симметричных направлений. Для направления [100] угол $\psi=0,\{J\}=\{100\}$, а компоненты групповой скорости $V_{g 3}^{\lambda}=V_{g z}^{\lambda}, V_{g 2}^{\lambda}=V_{g y}^{\lambda}, V_{g 1}^{\lambda}=V_{g x}^{\lambda}$. Для направления [110] угол $\psi=\pi / 4, \quad\{J\}=\{100\}$, а компоненты групповой скорости $V_{g 3}^{\lambda}=\left(-V_{g y}^{\lambda}+V_{g z}^{\lambda}\right) / \sqrt{2}, V_{g 2}^{\lambda}=\left(V_{g y}^{\lambda}+V_{g z}^{\lambda}\right) / \sqrt{2}$, $V_{g 1}^{\lambda}=V_{g x}^{\lambda}$. Для направления [111] угол $\psi=\operatorname{arctg} \sqrt{2}$, $\{J\}=\{110\}, \quad$ а компоненты групповой скорости определяются выражениями $V_{g 3}^{\lambda}=\left(V_{g x}^{\lambda}+V_{g y}^{\lambda}+V_{g z}^{\lambda}\right) / \sqrt{3}$, $V_{g 2}^{\lambda}=\left(V_{g x}^{\lambda}+V_{g y}^{\lambda}-2 V_{g z}^{\lambda}\right) / \sqrt{6}, V_{g 1}^{\lambda}=\left(V_{g x}^{\lambda}-V_{g y}^{\lambda}\right) / \sqrt{2}$. Для расчета второго эффекта Мак Карди [1] необходимо определить ориентационные параметры для градиента температуры вдоль направления [110] и двух вариантов ориентации боковых граней образца. В первом случае ориентация широкой грани образца $\{J\}=\{100\}$, узкой $-\{J\}=\{110\}, \quad$ поэтому компоненты $V_{g i}^{\lambda}$ определяются выражениями (6) при $\psi=\pi / 4$ :

$$
\begin{gathered}
V_{g 3}^{\lambda}=\left(-V_{g y}^{\lambda}+V_{g z}^{\lambda}\right) / \sqrt{2}, \quad V_{g 2}^{\lambda}=\left(V_{g y}^{\lambda}+V_{g z}^{\lambda}\right) / \sqrt{2}, \\
V_{g 1}^{\lambda}=V_{g x}^{\lambda} .
\end{gathered}
$$


Во втором случае ориентация широкой грани образца $\{J\}=\{110\}$, узкой $-\{J\}=\{100\}$, поэтому компоненты $V_{g i}^{\lambda}$ определяются выражениями (7) при $\psi=\pi / 2$ :

$$
\begin{gathered}
V_{g 3}^{\lambda}=\left(V_{g x}^{\lambda}+V_{g y}^{\lambda}\right) / \sqrt{2}, \quad V_{g 2}^{\lambda}=-V_{g z}^{\lambda}, \\
V_{g 1}^{\lambda}=\left(V_{g x}^{\lambda}-V_{g y}^{\lambda}\right) / \sqrt{2} .
\end{gathered}
$$

Итак, мы показали, что ориентационные параметры $[I(\psi)]$ и $\{J\}$ для произвольного направления теплового потока относительно осей кристалла могут быть определены через компоненты групповой скорости, параллельные и перпендикулярные тепловому потоку. Формулы (1) - (9) позволяют проанализировать эффекты Мак Карди в теплопроводности упруго анизотропных кристаллов в режиме кнудсеновского течения фононного газа.

Эффекты, обнаруженные в работе [1] для кристаллов $\mathrm{Si}$ и $\mathrm{CaF}_{2}$, имеют достаточно большую величину, поэтому представляет интерес рассчитать их значения для других упруго анизотропных кристаллов. Для анализа эффектов Мак Карди возьмем стержни с квадратным и прямоугольным сечениями тех же геометрических размеров, что и в работе [1], чтобы иметь возможность сравнить рассчитанные значения с экспериментально измеренными величинами эффектов для образцов $\mathrm{Si}$ и $\mathrm{CaF}_{2}$. Поэтому при анализе первого эффекта рассмотрим стержни с квадратным сечением, длиной $L=2.9$ см и со стороной $D=0.293 \mathrm{~cm}$, вырезанные с осями вдоль трех симметричных направлений - [100], [110] и [111]. Рассчитаем значения теплопроводности при $T=3 \mathrm{~K}$, как и в работе [1], когда фонон-фононные механизмы выморожены и доминирует диффузное рассеяние фононов на границах. Составим отношение теплопроводностей для симметричных направлений и определим величины первого эффекта Мак Карди для упруго анизотропных кристаллов: для кремния этот эффект, согласно [1], определяется отношениями $\kappa_{[001]}: \kappa_{[110]}: \kappa_{[111]} \approx 1.53: 1.08: 1$.

При анализе второго эффекта Мак Карди возьмем стержни с прямоугольным сечением длиной $L=5.5 \mu D_{1}$ и сторонами сечения $D_{1}=0.185, D_{2}=\mu D_{1}=0.638 \mathrm{~cm}$ $(\mu=3.45)$, вырезанные с осями вдоль направления [110] и ориентациями боковых граней $\{001\},\{110\}$. Ориентация широкой грани у одного из стержней будет $\{001\}$, а у другого $-\{110\}$. Составим отношение теплопроводностей для направления теплового потока [110] и двух ориентаций широких граней образца. Для кристаллов кремния при $T=3 \mathrm{~K}$ это отношение, определяющее величину второго эффекта Мак Карди, имеет вид $\kappa_{[011]}^{\{001\}}: \kappa_{[110]}^{\{011\}} \approx 1.33: 1$. Ориентации широких граней образца указаны верхним индексом в коэффициенте теплопроводности.

Далее мы проанализируем эффекты Мак Карди в кристаллах с различным типом анизотропии упругой энергии. Анализ уравнений Кристоффеля в работе [17] показал, что влияние анизотропии упругой энергии на спектр и вектора поляризации колебательных мод определяется безразмерным параметром $k-1=\left(c_{12}+2 c_{44}-c_{11}\right) /\left(c_{11}-c_{44}\right)\left(c_{i j}-\right.$ упругие модули второго порядка). В зависимости от знака параметра $k-1$, согласно [17], все кубические кристаллы могут быть разделены на кристаллы с положительной, $k-1>0$ (тип I, GaN, Ge, $\mathrm{Si}$, алмаз, $\mathrm{LiF}, \mathrm{GaSb}, \mathrm{GaAs}$ ), и отрицательной, $k-1<0$ (тип II, $\mathrm{CaF}_{2}, \mathrm{SrF}_{2}$ и $\mathrm{PbS}$ ), анизотропией упругих модулей второго порядка (см. таблицу). Для изотропных сред $k-1=0$. Как это видно из таблицы, соотношения величин, характеризующих эффекты Мак Карди в кристаллах с различным типом анизотропии упругой энергии, качественно различаются.

В строчках ${ }^{*} \mathrm{Si}$ и ${ }^{*} \mathrm{CaF}_{2}$ (в таблице) приведены экспериментальные данные первого эффекта Мак Карди [1] для образцов с квадратным сечением. Как видно из таблицы, рассчитанные значения первого и второго эффектов Мак Карди для $\mathrm{Si}$ и $\mathrm{CaF}_{2}$ согласуются с экспериментальными данными в пределах погрешности эксперимента [1]. Анизотропия теплопроводности в полупроводниковых кристаллах I и II типов качественно различается. Из таблицы видно, что для большинства наиболее актуальных и используемых в технических приложениях полупроводников I типа, таких как GaAs, $\mathrm{GaSb}, \mathrm{LiF}, \mathrm{InSb}, \mathrm{Ge}, \mathrm{Si}, \mathrm{HgSe}, \mathrm{MgO}$, при $T=3 \mathrm{~K}$ величины теплопроводности достигают максимума в направлениях типа [001] и оказываются на $50 \%$ больше минимальных значений в направлении [111]. Для кристаллов II типа ситуация обратная: величины теплопроводности достигают максимума в направлениях типа [111], а минимальных значений - в направлениях типа [001]. Для кристаллов $\mathrm{NaCl}, \mathrm{PbS}, \mathrm{SrF}_{2}$ величины теплопроводности в направлении [111] на $30 \%$, а для $\mathrm{CaF}_{2}$ на $40 \%$ больше, чем в направлении [001]. Таким образом, для значительного числа кристаллов с достаточно большим параметром $|k-1|$ анизотропия теплопроводности не является малой, и фокусировка фононов должна учитываться при анализе теплопроводящих свойств.

Что касается второго эффекта Мак Карди, то для кристаллов I типа GaN, GaAs, GaSb, LiF, InSb, Ge, $\mathrm{HgSe}, \mathrm{MgO}$ с теми же геометрическими параметрами образцов при $T=3 \mathrm{~K}$ он имеет такую же величину, как и для Si. Для направления градиента температуры [110] теплопроводность образцов с ориентаций широких граней $\{100\}$ оказывается на $30 \%$ больше, чем для образцов с ориентаций широких граней $\{110\}$. Однако в кристаллах II типа второй эффект Мак Карди качественно отличается. При направлении градиента температуры [110] теплопроводность образцов с ориентаций широких граней $\{100\}$ оказывается меньше, чем для образцов с ориентаций широких граней $\{110\}$. Для них величины, характеризующие второй эффект Мак Карди [1], заметно меньше: только для $\mathrm{CaF}_{2}$ и $\mathrm{SrF}_{2}$ его величины превышают $25 \%$, а для $\mathrm{NaCl}$ и $\mathrm{PbS}$ они $<20 \%$ (см. таблицу). Этот эффект также не является малым и должен учитываться при интерпретации экспериментальных данных. 
Отношения теплопроводностей для кубических кристаллов в режиме кнудсеновского течения фононного газа при $T=3 \mathrm{~K}$

\begin{tabular}{|c|c|c|c|c|c|c|c|c|}
\hline Тип & Кристалл & $\begin{array}{l}c_{11}, 10^{12} \\
\text { дин } / \mathrm{cm}^{2}\end{array}$ & $\begin{array}{l}c_{12}, 10^{12} \\
\text { дин/см }{ }^{2}\end{array}$ & $\begin{array}{l}c_{44}, 10^{12} \\
\text { дин } / \mathrm{cm}^{2}\end{array}$ & $k-1$ & $\begin{array}{c}\text { Первый } \\
\text { эффект } \\
\mu=1\end{array}$ & $\begin{array}{c}\text { Второй } \\
\text { эффект } \\
\mu=3.45\end{array}$ & $\begin{array}{l}\text { Второй } \\
\text { эффект } \\
\mu=10\end{array}$ \\
\hline \multirow[t]{12}{*}{ I } & $\mathrm{GaN}$ & 2.93 & 1.59 & 1.55 & 1.275 & $1.43: 1.07: 1$ & $1.28: 1$ & $1.49: 1$ \\
\hline & $\mathrm{Ge}$ & 1.289 & 0.483 & 0.671 & 0.87 & $1.49: 1.08: 1$ & $1.30: 1$ & $1.67: 1$ \\
\hline & $\mathrm{Si}$ & 1.677 & 0.65 & 0.804 & 0.67 & $1.50: 1.08: 1$ & $1.30: 1$ & $1.71: 1$ \\
\hline & ${ }^{*} \mathrm{Si}$ & 1.677 & 0.65 & 0.804 & 0.67 & $1.53: 1.07: 1$ & $1.33: 1$ & - \\
\hline & GaAs & 1.1904 & 0.5384 & 0.5952 & 0.90 & $1.51: 1.08: 1$ & $1.32: 1$ & $1.63: 1$ \\
\hline & $\mathrm{HgSe}$ & 0.69 & 0.51 & 0.23 & 0.61 & $1.52: 1.13: 1$ & $1.34: 1$ & $1.62: 1$ \\
\hline & $\mathrm{GaSb}$ & 0.885 & 0.404 & 0.433 & 0.85 & $1.51: 1.09: 1$ & $1.32: 1$ & $1.64: 1$ \\
\hline & $\mathrm{InSb}$ & 0.672 & 0.367 & 0.302 & 0.81 & $1.53: 1.09: 1$ & $1.33: 1$ & $1.62: 1$ \\
\hline & $\mathrm{LiF}$ & 1.246 & 0.424 & 0.649 & 0.78 & $1.48: 1.08: 1$ & $1.29: 1$ & $1.68: 1$ \\
\hline & $\mathrm{MgO}$ & 2.86 & 0.87 & 1.48 & 0.703 & $1.45: 1.07: 1$ & $1.26: 1$ & $1.63: 1$ \\
\hline & Алмаз & 10.76 & 1.25 & 5.76 & 0.40 & $1.20: 1.03: 1$ & $1.10: 1$ & $1.19: 1$ \\
\hline & YAG & 3.281 & 1.064 & 1.137 & 0.03 & $1.03: 1.01: 1$ & $1.02: 1$ & $1.03: 1$ \\
\hline \multirow[t]{7}{*}{ II } & $\mathrm{KCl}$ & 0.398 & 0.062 & 0.0625 & -0.63 & $1: 1.02: 1.08$ & $1: 1.02$ & $1: 1.00$ \\
\hline & $\mathrm{NaCl}$ & 0.575 & 0.099 & 0.133 & -0.48 & $1: 1.20: 1.32$ & $1: 1.18$ & $1: 1.27$ \\
\hline & $\mathrm{PbS}$ & 1.27 & 0.298 & 0.248 & -0.47 & $1: 1.19: 1.33$ & $1: 1.16$ & $1: 1.24$ \\
\hline & $\mathrm{CaF}_{2}$ & 1.74 & 0.56 & 0.359 & -0.33 & $1: 1.28: 1.42$ & $1: 1.27$ & $1: 1.45$ \\
\hline & ${ }^{*} \mathrm{CaF}_{2}$ & 1.74 & 0.56 & 0.359 & -0.33 & $1: 1.31: 1.44$ & - & - \\
\hline & $\mathrm{SrF}_{2}$ & 1.24 & 0.43 & 0.31 & -0.20 & $1: 1.23: 1.30$ & $1: 1.25$ & $1: 1.59$ \\
\hline & YIG & 2.69 & 1.077 & 0.764 & -0.04 & $1: 1.05: 1.06$ & $1: 1.04$ & $1: 1.06$ \\
\hline
\end{tabular}

Примечание. Первый эффект Мак Карди $[1],\left(\kappa_{[100]}^{\{100\}}: \kappa_{[110]}^{\{110\}}: \kappa_{[111]}^{\{110\}}\right)-$ для образцов с квадратным сечением $(L=2.9 \mathrm{~cm}, D=0.293$ см $) ;$ второй эффект Мак Карди [1], $\left(\kappa_{[110]}^{\{100\}}: \kappa_{[110]}^{\{110\}}\right)$ - для образцов с прямоугольным сечением $\left(L=5.5 \mu D_{1}, D_{1}=0.185 \mathrm{~cm}, D_{2}=\mu D_{1}=0.638 \mathrm{~cm}, \mu=3.45\right)$; ориентации широких граней $\{100\}$ и $\{110\}$ указаны в верхних индексах.

Проведенный анализ показал, что увеличение отношения ширины пластины к ее толщине (параметр $\mu$ ) приводит к увеличению второго эффекта Мак Карди. Поэтому в последнем столбце мы привели результаты расчета второго эффекта Мак Карди для образцов с прямоугольным сечением $\left(L=5.5 \mu D_{1}, D_{1}=0.185 \mathrm{~cm}\right.$, $\left.D_{2}=\mu D_{1}, \mu=10\right)$. Как видно из таблицы, второй эффект увеличился примерно в 2 раза: для кристаллов $\mathrm{InSb}, \mathrm{MgO}, \mathrm{GaAs}, \mathrm{GaSb}, \mathrm{Ge}, \mathrm{LiF}, \mathrm{Si}$ эффект возрастает от 63 до 71\% соответственно (см. таблицу). Приведенные выше результаты характеризуют роль фокусировки фононов в анизотропии теплопроводности. Можно надеяться, что они будут полезны не только при исследовании процессов теплопереноса в упругоанизотропных кристаллах, но и в технических приложениях. Одним из таких приложений может являться контроль за степенью совершенства обработки поверхности образцов с прямоугольным сечением. Поскольку наша теория количественно описывает теплопроводность при диффузном рассеянии фононов, то из сравнения данных эксперимента и теоретических расчетов появляет- ся возможность определения параметров зеркальности отражения фононов при различных способах обработки поверхности.

\section{3. Физическая интерпретация эффектов Маккарди в теплопроводности кубических кристаллов}

Рассмотрим влияние фокусировки на распространение и граничное рассеяние фононов в монокристаллических образцах конечных размеров и дадим наглядное физическое объяснение эффектам Мак Карди. Как уже отмечалось в [1-4], влияние фокусировки связано с различием направлений волнового вектора и групповой скорости, которая определяет направление распространения фононов (см. рис. 1). Проиллюстрируем этот эффект на примере моды $t_{2}$ в кристаллах $\mathrm{Si}$ для волновых векторов в плоскости $\{100\}$. Построим для нее изоэнергетическую поверхность (см. рис. 1,a). Групповая скорость 


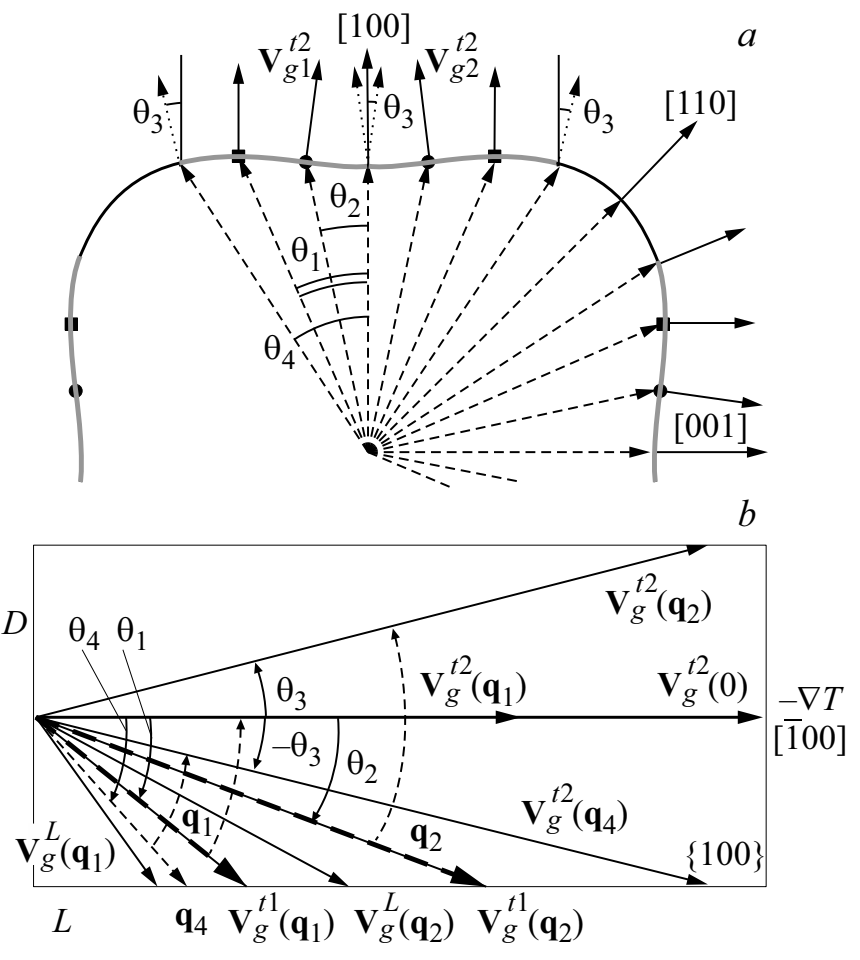

Рис. 1. Схема, иллюстрирующая фокусировку медленных поперечных мод в кристаллах $\mathrm{Si}$ для сечения изоэнергетической поверхности плоскостью $\{100\}$ (стрелками изображены волновые вектора внутри поверхности и соответствующие им групповые скорости фононов вне ее) (a) и влияние фокусировки на распространение фононов в образцах $\mathrm{Si}$ длиной $L$ с квадратным сечением $D \times D$ для волновых векторов в плоскости $\{100\}$ с углами $\theta_{1}=23.6^{\circ}, \theta_{2}=11.9^{\circ}, \theta_{3}=6.8^{\circ}$, $\theta_{4}=28.6^{\circ}$ и градиентом температуры вдоль [100] $(b)$.

фонона, определяющая направление переноса энергии, перпендикулярна этой поверхности. Из рис. 1 видно, что фокусировка фононов характеризуется углами $\theta_{1}, \theta_{2}, \theta_{3}$ и $\theta_{4}[18,19]$. Углы $\pm \theta_{1}$ определяют направления волнового вектора, для которого вектор групповой скорости параллелен направлению фокусировки. Углы $\pm \theta_{2}$ задают направления волновых векторов к точкам нулевой кривизны на изоэнергетической поверхности (см. рис. 1). Углы $\pm \theta_{3}$ определяют направления групповых скоростей в точках нулевой кривизны. Углы $\pm \theta_{4}$ определяют величину сектора волновых векторов, в которых групповые скорости будут коллинеарны их направлениям в точках нулевой кривизны, и тем самым они определяют область фокусировки фононов $[18,19]$.

В изотропной среде направление распространения фонона и его волнового вектора совпадают, и фононы с волновыми векторами в секторе $-\theta_{4} \leq \theta \leq \theta_{4}$ будут распространяться в том же самом секторе. Однако, как видно из рис. 1, направления волновых векторов и групповых скоростей отличаются, и фононы медленной поперечной моды в кремнии будут распространяться в существенно меньшем секторе, $-\theta_{3} \leq \theta \leq \theta_{3}$, который определяется направлениями групповых скоростей в точках нулевой кривизны на изоэнергетической поверхности (см. подробнее $[18,19])$. В результате, во-первых, в секторе фокусировки $-\theta_{3} \leq \theta \leq \theta_{3}$ средняя плотность состояний моды $t_{2}$ для $\mathrm{Si}$ будет в отношении $\theta_{4} / \theta_{3} \approx 4.2$ больше, чем в изотропной среде $\left(\theta_{4}=28.6^{\circ}, \theta_{3}=6.8^{\circ}\right)$. Напротив, для сектора дефокусировки $\pi / 2-\theta_{3} \geq \theta \geq \theta_{3}$ она будет в 2.3 раза меньше, чем в изотропной среде. В итоге в секторе фокусировки средняя плотность состояний моды $t_{2}$ будет в 9.9 раза больше, чем в секторе дефокусировки (см. рис. 1). Во-вторых, поскольку $\theta_{3} \ll \theta_{4}$, фононы будут отклоняться от боковых граней к оси стержня и распространяться в меньшем угловом секторе, то длины свободного пробега для них значительно возрастают (см. рис. 1). Заметим, что при диффузном рассеянии длина свободного пробега фонона с волновым вектором q определяется расстоянием, пройденным фононом до столкновения с поверхностью образца. Можно отметить некоторые особенности распространения фононов моды $t_{2}$ в плоскости $\{100\}$, обусловленные влиянием фокусировки. Во-первых, фононы с волновыми векторами $\mathbf{q}_{1}$ (для $\mathrm{Si} \theta_{1}=23.6^{\circ}$ ) будут распространяться в направлении теплового потока [100] (см. рис. 1). Их длина пробега при диффузном отражении от границ может быть ограничена либо объемными механизмами релаксации, либо длиной образца. Фононы с волновым вектором $\mp \mathbf{q}_{4}$ будут распространяться под существенно меньшим углом $\theta_{3}$, в результате их длина пробега возрастет (см. рис. 1).

Для продольной моды направление [001] является направлением дефокусировки. Поэтому продольные фононы будут отклоняться от оси образца в сторону боковой грани, и их длина свободного пробега будет меньше, чем в модели изотропной среды (см. рис. 1). Для волновых векторов в плоскости $\{100\}$ быстрая поперечная мода изотропна, эффект фокусировки для нее отсутствует. Ее длина свободного пробега будет совпадать с полученной в модели изотропной среды (см. рис. 1). Таким образом, из схем, приведенных на рис. 1, следует, что максимум теплопроводности для кристаллов I типа в направлениях [100] обусловлен вкладом медленных поперечных фононов.

Рассмотрим влияние фокусировки на распространение фононных мод в осевом сечении образца $\{100\}$ в случае, когда градиент температуры направлен вдоль [110]. Направление [110] соответствует дефокусировке медленной поперечной моды $t_{2}$. Поэтому фононы этой моды будут отклоняться в сторону грани образца и их длина свободного пробега будет меньше, чем в модели изотропной среды (см. рис. 1). Для волновых векторов в плоскости $\{100\}$ быстрая поперечная мода изотропна, эффект фокусировки для нее отсутствует $[18,19]$. Ее длина свободного пробега будет совпадать с полученной в модели изотропной среды. Продольные фононы в этой плоскости имеют локальную фокусировку в направлении [110]. Они будут отклоняться к оси образца, и их длина пробега будет больше, чем в модели изотропной среды. Следует отметить, что роль продольных фононов 
в фононном транспорте для кремния мала, поскольку для них теплоемкость на порядок меньше суммарной теплоемкости поперечных фононов, а вклад в теплопроводность для направлений [001] и [110] составляет всего 8 и $15 \%$ соответственно.

В направлении [111] фокусируются продольные фононы, их длина свободного пробега увеличивается почти в 2 раза по сравнению с направлением дефокусировки [001] и принимает максимальное значение (см. [6]). В этом направлении быстрая поперечная мода дефокусируется, и длины пробега фононов этой моды будут меньше, чем в модели изотропной среды. Фононы медленной поперечной моды в этом направлении имеют локальный максимум фокусировки, и длины пробега для них незначительно превосходят величины для модели изотропной среды [6]. Поскольку теплоемкость продольных фононов мала, то полная теплопроводность и средняя длина пробега фононов в направлении [111] принимают минимальное значение [6].

Непосредственный расчет длин свободного пробега в $\mathrm{Si}$ подтверждает приведенные выше качественные рассуждения (см. [6]). В работах [6,15] было показано, что длины свободного пробега для каждой колебательной моды достигают максимальных значений в направлениях фокусировки, причем в этих направлениях они превосходит длины пробега остальных колебательных мод. Их минимальные значения достигаются в направлениях дефокусировки, причем они оказываются меньше рассчитанных в модели изотропной среды. Так, например, в кристаллах $\mathrm{Si}$ длина свободного пробега моды $t_{2}$ в направлениях фокусировки превосходит длины пробега быстрой поперечной и продольной мод в 1.6 и 2.4 раза соответственно. Поскольку средняя плотность состояний этой моды в секторе фокусировки также возрастает, в результате действия этих двух эффектов вклад медленных поперечных фононов в теплопроводность для направления [100] при температуре $T=3 \mathrm{~K}$ достигает $61 \%$ полной теплопроводности и в 2 раза превышает вклад быстрой поперечной моды. В работах [6] показано, что для симметричных направлений результаты расчета длин пробега и теплопроводности хорошо согласуются с экспериментальными данными [1]. Итак, первый эффект Мак Карди в образцах $\mathrm{Si}$, а также в остальных кристаллах первого типа обусловлен медленной поперечной модой, которая фокусируется в направлении [001] и обеспечивает максимум теплопроводности в этом направлении.

Аналогичным образом можно построить изоэнергетические поверхности для кристалла $\mathrm{CaF}_{2}$ и рассмотреть влияние фокусировки на распространения фононов в кристаллах II типа с квадратным сечением. Поскольку этот анализ во многом повторяет приведенный выше для кристаллов кремния, то мы не будем воспроизводить его, а приведем сразу результаты расчета длин пробега фононов в кристаллах $\mathrm{CaF}_{2}$ (см. рис. 2). Как видно из рис. 2, в кристаллах второго типа первый эффект Мак Карди также обусловлен медленной поперечной

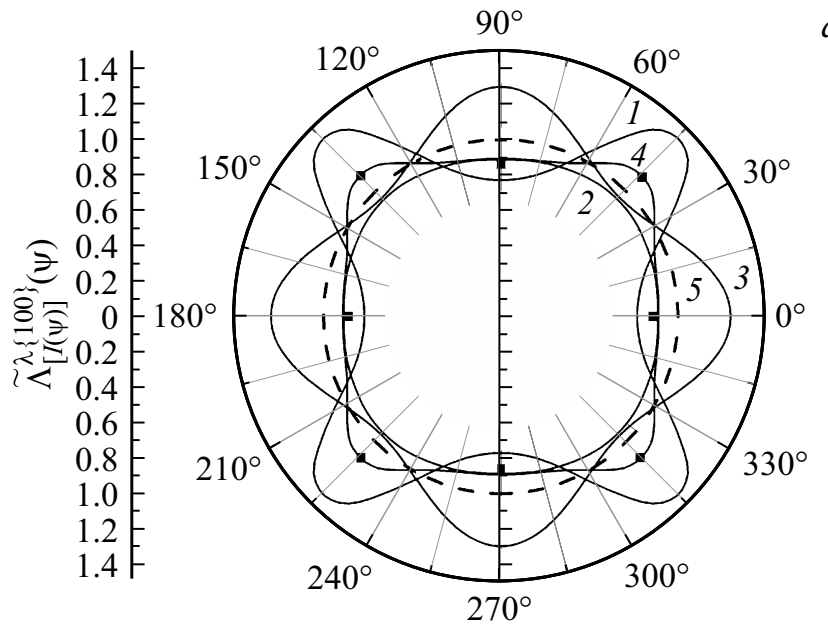

$a$

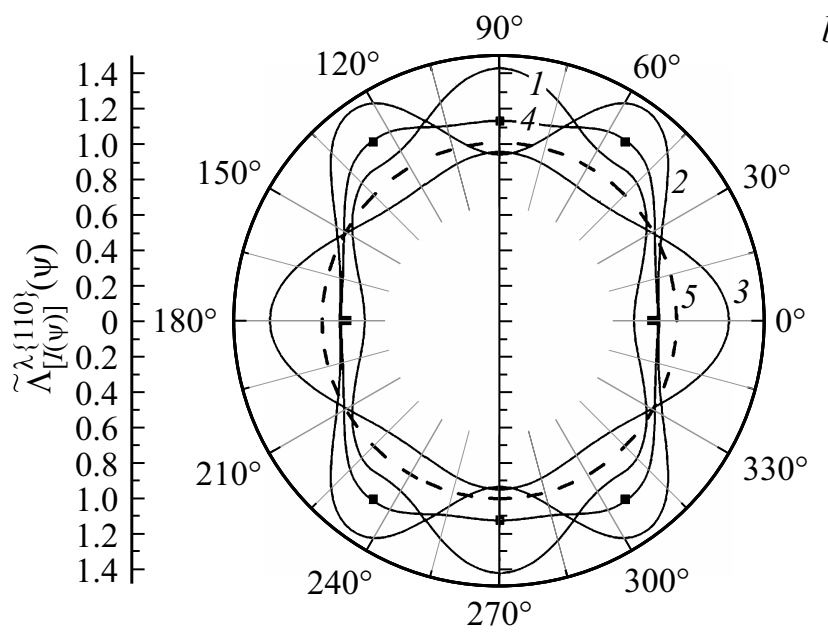

$b$

Рис. 2. Угловые зависимости приведенных длин свободного пробега $\tilde{\Lambda}_{[I(\psi)]}^{\lambda\{J\}}(\psi)=\Lambda_{[I(\psi)]}^{\lambda\{J\}} / D$ в кристаллах $\mathrm{CaF}_{2}$ для образцов длиной $L=2.9 \mathrm{~cm} \mathrm{с} \mathrm{квадратным} \mathrm{сечением} \mathrm{с} D=0.293 \mathrm{~cm}$ для случаев, когда градиент температур вращается в плоскости грани куба $\{J\}=\{100\}(a)$, в диагональной плоскости $\{J\}=\{110\}(b): 1-$ быстрая поперечная мода, $2-$ медленная поперечная мода, 3 - продольная мода, $4-$ средняя длина свободного пробега, 5 - длина свободного пробега в модели изотропной среды. Точки - экспериментальные данные [1].

модой, которая фокусируется и обеспечивает максимум теплопроводности в направлениях типа [111]. Ее длина свободного пробега в направлениях [111] превосходит длины пробега быстрой поперечной и продольной мод в 1.4 и 1.7 раза соответственно. Благодаря эффекту фокусировки фононов вклад медленных поперечных фононов в теплопроводность для направления [111] при температуре $T=3 \mathrm{~K}$ достигает $57 \%$ полной теплопроводности, тогда как вклады быстрой поперечной моды и продольных фононов составляют 34 и $8 \%$ соответственно. Результаты расчета хорошо согласуются с экспериментальными данными для симметричных направлений [1] (см. рис. 2). 

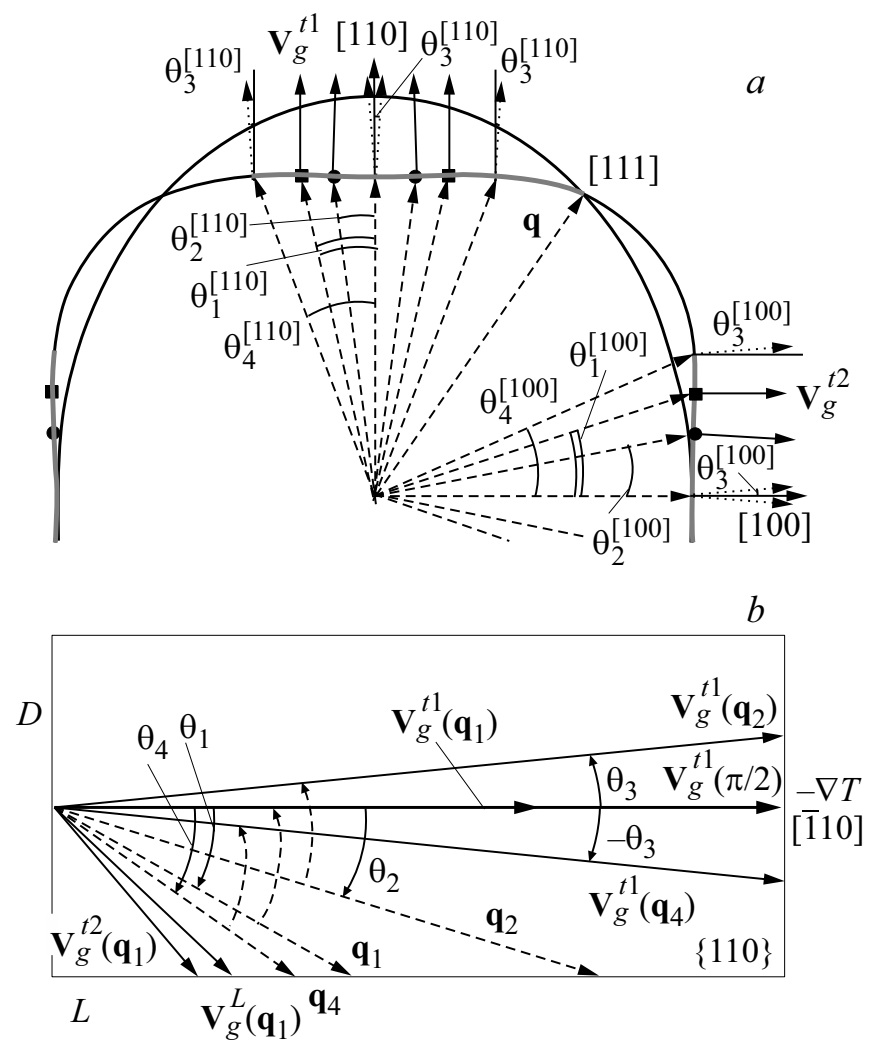

Рис. 3. Схема, иллюстрирующая фокусировку быстрых поперечных мод $(a)$ и влияние фокусировки на распространение фононов в образцах $\mathrm{Si}$ длиной $L$ с квадратным сечением $D \times D$ для волновых векторов в плоскости $\{110\}$ с углами $\theta_{1}=13.4^{\circ}$, $\theta_{2}=7.5^{\circ}, \theta_{3}=0.86^{\circ}, \theta_{4}=15.7^{\circ}$, градиентом температуры вдоль [110] (b).

Далее рассмотрим фононный транспорт в образцах $\mathrm{Si}$ с прямоугольным сечением и дадим физическое объяснение второго эффекта Мак Карди. Из работ Фукса [20], Зондгеймера [21] известно, что теплосопротивление таких образцов (как и пленок) определяется главным образом рассеянием фононов на широких гранях. Проанализируем влияние фокусировки на распространение и граничное рассеяние фононов в образцах с прямоугольным сечением и покажем, что зависимость теплопроводности от ориентации боковых граней образца обусловлена не различной рассеивающей способностью, как предполагалось в [16], а влиянием фокусировки на распространение и граничное рассеяние фононов в образцах с прямоугольным сечением. Рассмотрим сначала образцы с широкой гранью $\{001\}$ и узкой $\{110\}$. Поскольку основной вклад в граничное рассеяние фононов вносит рассеяние на широких гранях образца, проанализируем распределение теплового потока по поперечному сечению образца и влияние фокусировки на отклонение фононов различных поляризаций от направления градиента температуры [110] в плоскости $\{110\}$, перпендикулярной широкой грани образца (см. рис. 3). В направлении [110] фокусируются фононы быстрой поперечной моды, по- этому они будут отклоняться от широкой грани образца к направлению градиента температур (см. рис. 3). Их длина свободного пробега возрастет и будет больше, чем в модели изотропной среды. Из рис. 3 видно, что в образцах $\mathrm{Si}$ с прямоугольным сечением для теплового потока в направлении [110] фононы моды $t_{1}$ с волновыми векторами в секторе $-\theta_{4} \leq \theta \leq \theta_{4}$ будут отклоняться от боковых граней к оси стержня и распространяться в секторе $-\theta_{3} \leq \theta \leq \theta_{3}$. В результате для направлений [110] плотность состояний моды $t_{1}$ будет в 18.2 раза больше, а для направлений дефокусировки в 1.2 раза меньше, чем в изотропной среде [18]. При этом фононы моды $t_{1}$ в плоскости $\{110\}$ с волновым вектором $\mathbf{q}_{1}$ будут распространяться в направлении теплового потока [110], а фононы с волновым вектором $\mathbf{q}_{2}$ будут распространяться под углом $-\theta_{3}$ (см. рис. 3). Итак, для ориентации широкой грани $\{001\}$ длины пробега в кристаллах I типа благодаря эффекту фокусировки могут существенно возрасти по сравнению с моделью изотропной среды.

Направление [110] является направлением дефокусировки медленной поперечной моды, и ее длина свободного пробега меньше, чем в изотропной среде. Продольные фононы фокусируются в направлении [111], а направление [110] соответствует направлению дефокусировки для волновых векторов в плоскости $\{110\}$. Поэтому они будут отклоняться от направления градиента температур к широкой грани образца, их длина свободного пробега будет меньше, чем для изотропной среды. Как уже отмечалось, роль продольных фононов в фононном транспорте мала в связи с малой теплоемкостью.

Другая ситуация складывается для образцов с широкой гранью $\{110\}$ и узкой $\{001\}$. Аналогично предыдущему случаю мы должны рассматривать фокусировку фононов в плоскости, перпендикулярной широкой грани, т.е. в $\{001\}$. В плоскости $\{001\}$ быстрая поперечная мода изотропна, эффект фокусировки для нее отсутствует и длина свободного пробега для нее будет совпадать с полученной в модели изотропной среды. В этой плоскости, как и в плоскости $\{110\}$ для направления [110], медленная поперечная мода $t_{2}$ дефокусируется (см. рис. 1). Поэтому фононы этой моды будут отклоняться от направления градиента температуры в сторону широкой грани и их длина свободного пробега, как и в предыдущем случае, будет меньше, чем в модели изотропной среды. Продольные фононы в этой плоскости имеют локальную фокусировку в направлении [110], и они будут отклоняться от широкой грани образца к направлению градиента температур. Однако, как уже отмечалось выше, их роль в теплопроводности мала. Очевидно, что в рассматриваемом случае средняя длина свободного пробега и теплопроводность для образцов с широкой гранью $\{001\}$ будут больше, чем для образцов с широкой гранью $\{110\}$. Непосредственный расчет дает величину эффекта $30 \%$. Авторы [1] очень удачно выбрали направление $[110]$ для направления теплового потока, поскольку в этом направлении медленная поперечная 
мода в кристаллах I типа дефокусируется как в плоскости $\{001\}$, так и в плоскости $\{110\}$. Напротив, быстрая поперечная мода в плоскости $\{110\}$ фокусируется в направлении [110], а в плоскости $\{001\}$ для нее эффект фокусировки отсутствует. Итак, из приведенного выше анализа следует, что второй эффект Мак Карди [1] в кристаллах I типа обусловлен фокусировкой быстрой поперечной моды в направлении [110] для волновых векторов в диагональной плоскости.

Аналогично рассмотренному выше можно показать, что второй эффект Мак Карди в кристаллах II типа также обусловлен фокусировкой быстрой поперечной моды в направлении [110], но для волновых векторов не в плоскости $\{110\}$, как это имеет место в кристаллах I типа, а в плоскости грани куба. Построив изоэнергетические поверхности для кристалла $\mathrm{CaF}_{2}$, нетрудно убедиться, что области с отрицательной кривизной на этих изоэнергетических поверхностях в окрестности направлений [110] будут иметь место только в плоскости $\{001\}$, тогда как в плоскости $\{110\}$ она является выпуклой [19]. Поэтому наиболее сильно эффект фокусировки для длин свободного пробега и плотности фононных состояний будет выражен в окрестности направлений [110] в плоскости $\{001\}$. Фононы быстрой поперечной моды благодаря фокусировке будут отклоняться от широких граней пластины с ориентацией $\{011\}$ к оси пленки, тем самым увеличивая длины свободного пробега фононов. Итак, механизм второго эффекта Мак Карди в кристаллах II типа является тем же самым, что и в кристаллах I типа, и обусловлен быстрой поперечной модой. Однако в кристаллах II типа теплопроводность образцов с широкой гранью $\{001\}$ будет меньше, чем образцов с широкой гранью $\{110\}$.

\section{4. Заключение}

Рассчитаны эффекты Мак Карди в теплопроводности упруго анизотропных кристаллов в режиме кнудсеновского течения фононного газа. Показано, что в этом режиме использование трех модулей упругости для кубических кристаллов оказывается вполне достаточным, чтобы определить зависимости теплопроводности монокристаллических образцов от геометрических параметров и направлений теплового потока относительно кристаллографических осей без использования подгоночных параметров.

Рассмотрено влияние фокусировки на граничное рассеяние и распространение фононов в монокристаллических образцах с квадратным и прямоугольным сечениями и дано физическое объяснение эффектов Мак Карди. Показано, что первый эффект в образцах с квадратным сечением обусловлен медленной поперечной модой, фокусировка которой обеспечивает максимум теплопроводности в кристаллах обоих типов. В кристаллах I типа ее фокусировка и максимум теплопроводности достигаются в направлении [001], тогда как в кристаллах II типа фоку- сировка медленной моды и максимум теплопроводности достигаются в направлении [111].

Исследование влияния фокусировки на граничное рассеяние в образцах с прямоугольным сечением показало, что второй эффект Мак Карди обусловлен изменением распределения теплового потока по поперечному сечению образца благодаря фокусировке быстрой поперечной моды в кристаллах обоих типов. Этот эффект качественно отличается для кристаллов с различным типом анизотропии упругой энергии. В кристаллах I типа теплопроводность для образцов с широкой гранью $\{001\}$ будет больше, чем для образцов с широкой гранью $\{110\}$, тогда как в кристаллах II типа наоборот: теплопроводность для образцов с широкой гранью $\{001\}$ будет меньше, чем для образцов с широкой гранью $\{110\}$. Проведенный анализ показал, что увеличение отношения ширины пластины к ее толщине от значения $\mu=3.45$ до $\mu=10$ приводит к увеличению второго эффекта Мак Карди более, чем в 2 раза. При этом зависимость теплопроводности от ориентации плоскости пластинки может стать более существенной, чем ее зависимость от направлений теплового потока относительно кристаллографических осей. Несомненно, что полученные в работе результаты представляют интерес для широкого круга исследователей и могут быть использованы в технических приложениях.

Работа выполнена в рамках государственного задания по теме „Спин“ АAAA-A18-118020290104-2 при поддержке проекта № 32-1.1.3.5 Программы фундаментальных исследований Президиума РАН согласно контракту Минобрнауки № 14.Z50.31.0025.

\section{Список литературы}

[1] A.K. Mc Curdy, H.J. Maris, C. Elbaum. Phys. Rev. B, 2, 4077 (1970).

[2] B. Taylor, H.J. Maris, C. Elbaum. Phys. Rev. Lett., 23, 416 (1969).

[3] H.J. Maris. J. Acoust. Soc. Am., 50, 812 (1971).

[4] J.P. Wolfe. Imaging Phonons Acoustic Wave Propagation in Solids (Cambridge University Press, N. Y., 1998).

[5] H.B.G. Casimir. Physica, 5, 495 (1938).

[6] И.И. Кулеев, И.Г. Кулеев, С.М. Бахарев, А.В. Инюшкин. ФТT, 55, 24 (2013).

[7] I.I. Kuleyev, I.G. Kuleyev, S.M. Bakharev, A.V. Inyushkin. Phys. Status Solidi B, 251, 991 (2014).

[8] I.I. Kuleyev, I.G. Kuleyev, S.M. Bakharev, A.V. Inyushkin. Physica B, 416, 81 (2013).

[9] H.J. Maris, S. Tamura. Phys. Rev. B, 85, 054304 (2012).

[10] N. Mingo. Phys. Rev. B, 68, 113308 (2003).

[11] Y.F. Zhu, J.S. Lian, Q. Jiang. Appl. Phys. Lett., 92, 113101 (2008).

[12] D.G. Cahill, W.K. Ford, K.E. Goodson, G.D. Mahan, A. Majumdar, H.J. Maris. J. Appl. Phys., 93, 793 (2003).

[13] A.D. Mc Connell, K.E. Goodson. Ann. Rev. Heat Transfer, 14, 129 (2005).

[14] D.G. Cahill, P.V. Braun, G. Chen, D.R. Clarkce, S. Fan, K.E. Goodson. J. Appl. Phys. Rev., 1, 011305 (2014). 
[15] И.И. Кулеев, И.Г. Кулеев, С.М. Бахарев. ЖЭТФ, 146, 525 (2014).

[16] Z. Aksamija, I. Knezevic. Phys. Rev. B, 82, 045319 (2010).

[17] И.Г. Кулеев, И.И. Кулеев. ФТТ, 49, 422 (2007).

[18] И.И. Кулеев, С.М. Бахарев, И.Г. Кулеев, В.В. Устинов. ФМM, 118, 12 (2017).

[19] I.I. Kuleyev, I.G. Kuleyev, S.M. Bakharev. Phys. Status Solidi C, 14, 1600263 (2017).

[20] K. Fuchs. Proc. Cambridge Philos. Soc., 34, 100 (1938).

[21] E.H. Sondheimer. Adv. Phys., 1, 1 (1952).

Редактор Л.В. Шаронова

\section{Mc Curdy's effects in thermal conductivity of the elastically anisotropic crystals in the Knudsen phonon gas flow regime}

I.I. Kuleyev, I.G. Kuleyev, S.M. Bakharev

Institute of Metal Physics,

Ural Branch of the Russian Academy of Sciences, 620990 Yekaterinburg, Russia

Abstract The influence of phonon focusing on the phonon transport in semiconductor and dielectric crystals with different types of anisotropy of elastic energy at low temperatures has been investigated. Mc Curdy's effects in thermal conductivity of the elastically anisotropic crystals in the Knudsen phonon gas flow regime has been calculated. The influence of the phonon focusing on the propagation and the phonon boundary scattering in samples with square and rectangular cross sections has been analyzed. A physical explanation of the Mc Curdy's effects has been given. 